\title{
Biomarkers for Alzheimer's disease: from pathogenesis to drug development
}

\author{
Kenji Hashimoto ${ }^{1}$
}

Published online: 12 June 2018

○) Springer-Verlag GmbH Germany, part of Springer Nature 2018

At present, five drugs have been approved for the treatment of Alzheimer's disease (AD), including four cholinesterase inhibitors (tacrine, donepezil, rivastigmine, and galantamine) and one $N$-methyl-D-aspartate receptor (NMDAR) antagonist memantine. However, no new drugs for $\mathrm{AD}$ have been approved since 2003. According to the Cleveland Clinical study, $99.6 \%$ of clinical trials of AD candidate drugs between 2002 and 2012 were failures. The majority of these candidate drugs were symptomatic agents aimed at improving cognition (36.6\%), followed by disease-modifying small molecules (35.1\%) and disease-modifying immunotherapies (18\%) [1]. Many trials fail because the candidates are unable to show a drug/placebo difference or have unacceptable toxicity [1]. The reasons for the lack of efficacy of these $\mathrm{AD}$ candidates should be evaluated to improve the success rate for future drug development. More precise understanding of the complex pathogenesis of AD may provide new approaches to novel AD drugs. Furthermore, development of novel $\mathrm{AD}$ biomarkers may improve early diagnosis in routine clinical practice and research.

NMDAR antibodies play a role in neurodegenerative disorders [2]. In this issue, Busse et al. [3] measured the presence of NMDAR antibodies in serum and cerebrospinal fluid (CSF) in patients with mild cognitive impairment $(n=59)$ and different types of dementia, $\mathrm{AD}(n=156)$, subcortical ischemic vascular dementia (SIVD: $n=61$ ), and frontotemporal dementia (FTD: $n=34$ ). Serum IgA/IgM NMDAR antibodies and/or a disturbed blood-CSF barrier were sporadically present in all of the investigated patient groups. In AD patients, these antibodies often developed during the disease course. Loss of function of the blood-CSF barrier was associated with a progressive decline in cognitive

Kenji Hashimoto

hashimoto@faculty.chiba-u.jp

1 Division of Clinical Neuroscience, Chiba University Center for Forensic Mental Health, 1-8-1 Inohana, Chiba 260-8670, Japan function and a poor prognosis. The data showed that NMDAR antibodies are present in serum from patients with different types of dementia as well as from healthy elderly individuals. Although the origin and biological impact of NMDAR antibodies remain unresolved, the origin of these antibodies seems to be in the periphery because NMDARs are expressed in peripheral tissues [4].

Monocytes and macrophages, which are tissue-specific monocytic cells such as microglia in the brain, are an important part of the innate immune system. AD patients are diagnosed to have an increased number of quinolinic acid (an endogenous NMDAR agonist)-positive cells in their peripheral monocytes compared with age-matched controls [5]. Furthermore, these cell populations remain increased in $\mathrm{AD}$ for up to 1 year after treatment initiation with the $\mathrm{AD}$ drug rivastigmine; no alterations were detected in healthy elderly subjects. Quinolinic acid is suggested to play a role in the neurotoxicity of AD via several mechanisms. Quinolinic acid directly acts as an agonist at NMDARs, inducing NMDAR-mediated excitotoxicity in the brain. Furthermore, it accumulates in amyloid- $\beta$ plaques and within dystrophic neurons, inducing tau phosphorylation through NMDAR activation. Collectively, it is likely that quinolinic acidexpressing cells in peripheral monocytes contribute to the neurotoxicity of quinolinic acid in the brain [5].

Cholesterol, an essential component in the structure and function of cell membranes, is also associated with AD pathogenesis [6]. Previous studies have demonstrated a decrease in 24(S)-hydroxycholesterol and an increase in 27(S)-hydroxycholesterol in AD patients [7]. Interestingly, there is considerable evidence suggesting a relationship between the degree of brain atrophy and plasma levels of the cholesterol metabolite 24(S)-hydroxycholesterol [7]. In this issue, Costa et al. [8] found decreased plasma levels of 24(S)-hydroxycholesterol in probable AD patients $(n=30)$ compared with healthy controls $(n=33)$, whereas plasma levels of 27(S)-hydroxycholesterol and total cholesterol were not altered. Furthermore, the presence of an ApoE4 
allele was not associated with cholesterol, 24(S)-hydroxycholesterol or 27(S)-hydroxycholesterol. After 3 months of donepezil treatment, there was no change in cholesterol or 24(S)-hydroxycholesterol level, which remained lower than in controls; however, 27(S)-hydroxycholesterol levels did show a slight increase. After 6 months of treatment, there was a discrete increase in cholesterol levels, but no change in 24(S)-hydroxycholesterol levels. Although Costa et al. [8] suggest a role of disturbed cholesterol turnover in AD pathogenesis, further studies using larger populations are needed.

A recent meta-analysis demonstrated that several core biomarkers can differentiate AD patients from controls with good performance, namely CSF total tau, phosphorylated tau, and amyloid- $\beta_{42}$. CSF neurofilament light protein (NFL) and plasma total tau also had large effect sizes when differentiating between controls and AD patients [9]. Soluble amyloid precursor protein $\beta$ (sAPP $\beta$ ), a potential plasma biomarker candidate for $\mathrm{AD}$, is a product of the initial phase of the amyloid cascade. In this issue, Alexopoulos et al. [10] reported that plasma levels of sAPP $\beta$ were significantly lower in patients with symptomatic AD (21 with mild cognitive impairment due to $\mathrm{AD}$ and 44 with $\mathrm{AD}$ dementia) with AD-typical cerebral hypometabolic patterns compared with cognitively healthy elderly subjects $(n=27)$ without preclinical AD. In contrast, there were no differences in plasma levels of sAPP $\beta$ between MCI subjects and AD patients, although their sAPP $\beta$ levels were lower than those of healthy controls. This study suggests an association between the plasma sAPP $\beta$ levels and AD. It is thus likely that plasma sAPP $\beta$ levels could serve as a potential noninvasive and cost-effective biomarker of AD. Nonetheless, further studies using larger sample sizes are needed.

In conclusion, abnormalities in the brain-immune system, including NMDAR antibodies, quinolinic acid, cholesterol metabolism, and amyloid synthesis, play a key role in the pathogenesis of AD. Therefore, it is likely that early pharmaceutical intervention in combination with multiple diagnostic biomarkers may prevent or delay the onset of $\mathrm{AD}$.

Acknowledgements This study was partly supported by AMED under Grant Number JP18dm0107119.

\section{Compliance with ethical standards}

Conflict of interest The author declare that they have no conflict of interest.

\section{References}

1. Cummings JL, Morstorf T, Zhong K (2014) Alzheimer's disease drug-development pipeline: few candidates, frequent failures. Alzheimers Res Ther 6:37

2. Venkatesan A, Adatia K (2017) Anti-NMDA-receptor encephalitis: from bench to clinic. ACS Chem Neurosci 8:2586-2596

3. Busse M, Kunschmann R, Dobrowolny H, Hoffmann J, Bogerts B, Steiner J, Frodil T, Busse S (2018) Dysfunction of the bloodcerebrospinal fluid-barrier and $\mathrm{N}$-methyl-D-aspartate glutamate receptor antibodies in dementia. Eur Arch Psychiatry Clin Neurosci. https://doi.org/10.1007/s00406-017-0768-y

4. Bozic M, Valdivielso JM (2017) Functional distribution and regulation of the NMDAR in the kidney, heart and parathyroid gland. In: The NMDA receptors" edited by Kenji Hashimoto. Humana Press, New York, pp 51-68

5. Busse M, Hettler V, Fischer V, Mawrin C, Hartig R, Dobrowolny H, Bogerts B, Frodl T, Busse S (2018) Increased quinolinic acid in peripheral mononuclear cells in Alzheimer's dementia. Eur Arch Psychiatry Clin Neurosci. https://doi.org/10.1007/s0040 6-017-0785-y

6. Di Paolo G, Kim TW (2011) Linking lipids to Alzheimer's disease: cholesterol and beyond. Nat Rev Neurosci 12:284-296

7. Leoni V, Caccia C (2013) 24S-hydroxycholesterol in plasma: a marker of cholesterol turnover in neurodegenerative diseases. Biochimie 95:595-612

8. Costa AC, Joaquim HPG, Nunes VS, Kerr DS, Ferreira GS, Forlenza OV, Gattaz WF, Talib LL (2018) Donepezil effects on cholesterol and oxysterol plasma levels of Alzheimer's disease patients. Eur Arch Psychiatry Clin Neurosci. https://doi. org/10.1007/s00406-017-0838-2

9. Olsson B, Lautner R, Andreasson U, Öhrfelt A, Portelius E, Bjerke M, Hölttä M, Rosén C, Olsson C, Strobel G, Wu E, Dakin K, Petzold M, Blennow K, Zetterberg H (2016) CSF and blood biomarkers for the diagnosis of Alzheimer's disease: a systematic review and meta-analysis. Lancet Neurol 15:673-684

10. Alexopoulos P, Gleixner LS, Werle L, Buhl F, Thierjung N, Giourou E, Kagerbauer SM, Gourziz P, Kübler H, Grimmer T, Yakushev I, Martin J, Kurz A, Perneczky R (2018) Plasma levels of soluble amyloid precursor protein $\beta$ in symptomatic Alzheimer's disease. Eur Arch Psychiatry Clin Neurosci. https://doi. org/10.1007/s00406-017-0815-9 